\title{
Segmented road grade estimation for fuel efficient heavy duty vehicles
}

\author{
Per Sahlholm and Karl Henrik Johansson
}

\begin{abstract}
Long haulage road transport consumes considerable amounts of energy in today's world. Predictive control strategies based on digital maps can significantly lower the portion being wasted in traditional cruise control operated highway driving. Such control strategies rely on high quality stored road grade information. This paper describes a newly developed method to estimate the road grade using sensors commonly found on standard heavy duty vehicles. The method utilizes a piecewise linear road model derived from highway design methodologies. The estimation method has been implemented and evaluated experimentally, and is shown to give better results compared to an existing method.
\end{abstract}

\section{INTRODUCTION}

The large mass of a heavy duty vehicle (HDV) makes it very sensitive to the force of gravity in hilly terrain. In steep inclines the vehicle looses speed, and on extended downhill sections braking to avoid overspeeding becomes a necessity. If the road grade ahead is known it can be used as input to a control system that adjusts the speed of the vehicle to achieve increased energy efficiency. The energy efficiency of other on-board systems can also be improved, e.g. the gearshift strategy and scheduling of auxiliary unit duty cycles can be adjusted. The longitudinal road grade is thus an important property for achieving energy efficient control of HDVs. Knowledge of the road grade can come from many sources, but one attractive alternative would be to estimate the road grade using sensors already commonplace in production vehicles. Given that the estimated data can be stored on-board, and then retrieved when returning to the same road, no external data collection is required.

Highways are designed built according to specifications on, among other things, the vertical road grade profile. Based on these specifications it is possible to create a spatial model for changes in the road grade, and this model can be used for improved road grade estimation. In this paper background knowledge from the Swedish road administration on how the vertical curves of Swedish highways are constructed is used to derive an algorithm for on-board road grade estimation in HDVs. A key feature of the method is that measurements from subsequent runs along a road can easily be used to improve a stored estimate. The estimation performance of the proposed method is analyzed based on experimental results and compared to a previous method.

The authors gratefully acknowledge the support of the Swedish programme board for vehicle research through the IVSS programme.

P. Sahlholm is with Scania CV AB, SE-151 87 Södertälje, Sweden, and the ACCESS Linnaeus Center, KTH, SE-100 44 Stockholm, Sweden per.sahlholmescania.com

K.H. Johansson is with the ACCESS Linnaeus Center, KTH, SE-100 44 Stockholm, Sweden kallej@ee.kth.se

\section{A. Related Work}

A multitude of methods for estimating the road grade can be found in the literature. Methods for estimation of the instantaneous road grade in production vehicles generally include system cost as an important parameter. Contributions focused on mapping applications often utilize expensive hardware, that enables accurate results in a single pass over the road. The approach described herein, where many measurement from low-cost sensors are merged into a road grade map is quite rare.

A road grade sensor for automotive use is described in a patent application filed as early as 1971 [1]. More recent contributions often employ GPS receivers of various kinds to obtain road grade estimates [2]. In some cases driveline sensors are also used [3], [4]. On-line road grade estimation based on accelerometers, calculated driveline torque and a vehicle model, or other on board sensors, is state-of-theart in today's vehicles. These methods have the advantage of not needing any external signal, such as the GPS, but don't provide bias compensation or easy fusion of data from multiple runs along the road. One proposed method and a survey of the area can be found in [5].

This paper presents a study on what can be gained from using an improved road grade model together with data segmentation. This substantial contribution is compared with an earlier result. Our earlier contributions on road grade estimation can be found in [6], [7]

\section{B. Contribution}

This paper presents a method to estimate the road grade based on sensors that are standard mounted in HDVs, and background information on how highways are designed and built. It is known from road design practices that the broad character of highway road grades can be described by piecewise linear functions. Thus a three step estimation method is proposed. First the measured data is used to obtain an initial road grade estimate. In the second step linear segments are identified in the road grade signal. In the third step the linear segments are used with the measured data to produce a final road grade estimate. The whole process can be iteratively applied on successive passes over the same road section. The proposed method is implemented and evaluated experimentally. The performance of the new method is compared to that of the iterative road grade estimation method applied without the piecewise linear road model. The influence of the discretization step size is studied. 


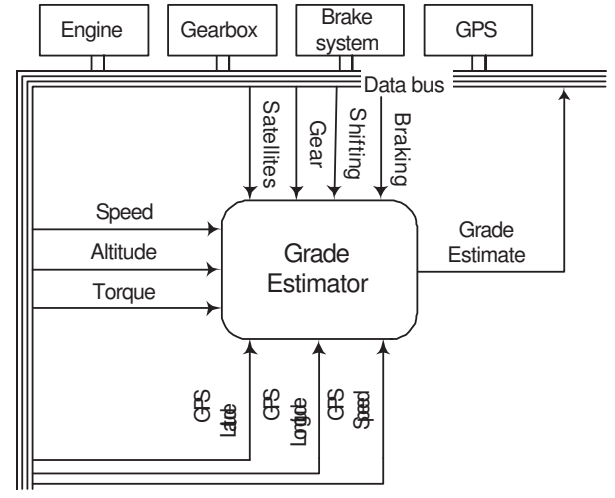

Fig. 1. The studied problem is how to estimate the road grade based on sensor information from a standard HDV, using the piecewise linear property of the road grade signal. The proposed estimator depends on two directly measured states; the vehicle speed and road altitude. The engine torque is treated as a measured input signal. The estimator also relies on auxiliary information about when braking and gearshifts occur, the currently engaged gear and the number of tracked GPS satellites.

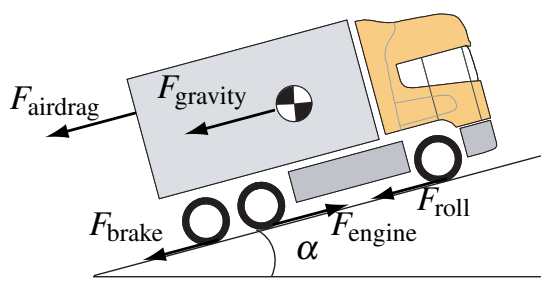

Fig. 2. Longitudinal forces acting on a vehicle traveling on a road with the road grade $\alpha$. The forces are generally time-varying; time has been left out of the equations for clarity.

\section{METHODOLOGY}

The proposed estimation method is based on vehicle and road models that are used to relate measured signals to estimated quantities. The vehicle model is derived from the laws of physics governing the longitudinal motion of a HDV. The road model is based on the design regulations governing the construction of highways in Sweden. The vehicle and road models are combined into a system model, that is used in a Kalman filter based method to arrive at a combined road grade estimate based on the current measurement and any pre-existing estimate stored in a map. Figure 1 gives an overview of the system architecture, with the measured signals. The road grade $\alpha$ is defined as the angle of the road plane in the longitudinal direction relative to a horizontal plane. This is often expressed in percent, calculated as climbed altitude divided by driven distance.

\section{A. Vehicle model}

A vehicle model is used to find the road grade from Newton's second law of motion. The main longitudinal forces acting on a HDV are considered, they are shown in Figure 2.

The forces shown in Figure 2 are modeled as follows. $F_{\text {engine }}=i_{\mathrm{t}} i_{\mathrm{f}} \eta_{\mathrm{t}} \eta_{\mathrm{f}} / r_{\mathrm{w}} \times T_{\mathrm{e}}$ is the net engine force, given by the transmission gear ratio $i_{\mathrm{t}}$ and efficiency $\eta_{\mathrm{t}}$, final gear ratio $i_{\mathrm{f}}$
TABLE I

MINIMUM CONVEX VERTICAL CURVE RADIUS $R$ FOR ROADS WITH AT LEAST TWO LANES, WITH REGARD TO SIGHT DISTANCE FOR PASSENGER CARS. HIGH, MEDIUM, AND LOW REFERS TO THE CHOSEN VISIBILITY STANDARD FOR THE ROAD. EXCERPT OF TABLE 11 - 1 IN [9].

\begin{tabular}{ccccc}
\hline \hline & & High & Medium & Low \\
$V_{\text {ref }}(k m / h)$ & Environment & $\mathrm{R}(\mathrm{m})$ & $\mathrm{R}(\mathrm{m})$ & $\mathrm{R}(\mathrm{m})$ \\
\hline 50 & Urban, main road & 1200 & 400 & 300 \\
70 & Countryside & 3000 & 1800 & 1200 \\
90 & Countryside & 7000 & 6000 & 5000 \\
110 & Countryside & 16000 & 13000 & 9000 \\
\hline \hline
\end{tabular}

and efficiency $\eta_{\mathrm{f}}$, and the wheel radius $r_{\mathrm{w}}$. The engine torque is denoted by $T_{\mathrm{e}} . F_{\text {airdrag }}=1 / 2 \times c_{\mathrm{w}} A_{\mathrm{a}} \rho_{\mathrm{a}} v^{2}$ is known through the measured vehicle speed $v$ and the air drag coefficient $c_{\mathrm{w}}$, vehicle frontal area $A_{\mathrm{a}}$, and air density $\rho_{\text {air. }}$. A very simple model $F_{\text {roll }}=m g c_{\mathrm{r}} \cos \alpha$ gives the rolling resistance from the vehicle mass $m$, gravity $g$, coefficient of rolling resistance $c_{\mathrm{r}}$, and road grade $\alpha$. The road grade also appears in the gravity induced force $F_{\text {gravity }}=m g \sin \alpha$. The brake force $F_{\text {brake }}$ is excluded from the model since it is generally unknown in a standard HDV, its influence is later considered by placing less trust in estimates when braking occurs. The total dynamic vehicle mass is expressed as $m_{\mathrm{t}}=J_{\mathrm{w}} / r_{\mathrm{w}}^{2}+m+i_{\mathrm{t}}^{2} i_{\mathrm{f}}^{2} \eta_{\mathrm{t}} \eta_{\mathrm{f}} J_{\mathrm{e}} / r_{\mathrm{w}}^{2}$ where $J_{\mathrm{w}}$ and $J_{\mathrm{e}}$ represent the inertia of the engine and the wheels respectively. This vehicle model is commonly used when a longitudinal model without the dynamic effects of various components in the driveline is desired. A thorough derivation can be found in [8].

\section{B. Road model}

Roads are built according to specifications determined by construction engineers. The specifications for Swedish roads give a good starting point for a model to describe highway road grades. The Swedish road authority has published a document [9], that lists requirements and guidelines for all road construction. The document states that the vertical road profile should be made up of segments with constant grade and vertical curves. The concave curves, going into more uphill gradients, are described by the parabola given by $\Delta z(\mathrm{~d})=\mathrm{d}^{2} / 2 \mathrm{R}$ where $\Delta z$ is the relative altitude, $\mathrm{d}$ is a horizontal distance measured relative to the lowest point of the parabola, and $R$ is a design parameter. The part of the parabola to use is determined by the road grades at the start and end of the vertical curve. For convex vertical curves, i.e. hilltops, the parabola is flipped upside down.

The value of the design variable $R$ depends on a number of factors, such as traffic safety, driving dynamics, visibility conditions, terrain and esthetics. The chosen vertical arc length and radius parameter have to match the surrounding terrain, and provide sufficient visibility for drivers to be able to stop before obstacles. For a major highway to be considered to have a good visibility standard when designed for a speed of $110 \mathrm{~km} / \mathrm{h}$ the minimum convex vertical curve radius, as listed in Table $\mathrm{I}$, is $R=16000 \mathrm{~m}$.

Assuming that the vertical road profile can only consist of 
segments with constant road grade, or parabolic segments as described above, we obtain a road model with the distance along the road as the independent variable. For the magnitude of road grades of interest $\alpha(\mathrm{d})=\arctan (\mathrm{dz} / \mathrm{dd}) \approx \mathrm{dz} / \mathrm{dd}=$ $\mathrm{d} / \mathrm{R}$. The change in $\alpha$ can thus be approximated as $d \alpha / d \mathrm{~d} \approx$ $1 / R$. Since the approximation of $d \alpha / d \mathrm{~d}$ does not depend on $\mathrm{d}$, the distance from the start of a local parabola, $\mathrm{d}$ can be replaced by $s$, the distance along the road. Let $\dot{\alpha}=d \alpha / d s$. Dividing the road into $N$ segments we then have local models $\dot{\alpha}_{i}(s)=d \alpha_{i} / d s= \pm 1 / R_{i}=c_{i}$ for convex and concave vertical curves between the knot points, with $i=1, \ldots, N$.

In this paper two different road grade models are used. Initially the assumption $c_{i}=0$ for all $i$ is used together with a corresponding process variance in the Kalman filter (low trust in the model) to generate a first estimate of the road grade. The estimated $\alpha(s)$ based on this assumption is then divided into $M$ piecewise linear segments by fitting a linear spline with a least squares cost function and unknown knot points to the road grade estimate obtained with the first model. Constrained non-linear optimization is used to find suitable knot points based on the associated least-squares spline interpolation error. Since the true number of segments $N$ is unknown, in general $M \neq N$. This model is subsequently used to obtain a new road grade estimate from the measured data.

\section{System model}

By combining the road and vehicle models with altitude information from a GPS receiver, denoted by $z$, a complete system model is obtained. Since the aim of this work is to estimate the road grade at locations along the road, the system model is expressed in a spatially sampled form. The spatial sampling distance depends on the desired resolution of the final road grade profile, and affects how the road grade model influences the results. This is further analyzed in Section III-C. The letter $k$ is used to indicate the distance index in the model.

In the system model the engine torque and the road grade model are regarded as input signals $u_{k}=\left[T_{\mathrm{e}_{k}} c_{i_{k}}\right]^{T}$. Together with the state vector $x=\left[\begin{array}{lll}v & z & \alpha\end{array}\right]^{T}$ this gives the spatially sampled system model

$$
\underbrace{\left[\begin{array}{c}
v_{k+1} \\
z_{k+1} \\
\alpha_{k+1}
\end{array}\right]}_{x_{k+1}}=\underbrace{\left[\begin{array}{c}
v_{k}+\Delta s \frac{d v_{k}}{d s} \\
z_{k}+\Delta s \sin \alpha_{k} \\
\alpha_{k}+\Delta s c_{i k}
\end{array}\right]}_{f_{k}\left(x_{k}, u_{k}\right)}+\underbrace{\left[\begin{array}{c}
w_{k}^{v} \\
w_{k}^{z} \\
w_{k}^{\alpha}
\end{array}\right]}_{w_{k}}
$$

where $w_{k}$ is process noise. The rate of change in speed is given by

$$
\begin{aligned}
\frac{d v_{k}}{d s}= & \frac{i_{\mathrm{t}} i_{\mathrm{f}} \eta_{\mathrm{t}} \eta_{\mathrm{f}}}{r_{\mathrm{w}} m_{\mathrm{t}}} \frac{T_{\mathrm{e} k}}{v_{k}}-\frac{\frac{1}{2} c_{\mathrm{d}} A_{\mathrm{a}} \rho_{\mathrm{a}}}{m_{\mathrm{t}}} v_{k} \\
& -\frac{m g}{m_{\mathrm{t}}} \frac{1}{v_{k}}\left(c_{\mathrm{r}} \cos \alpha_{k}+\sin \alpha_{k}\right)
\end{aligned}
$$

Two states, $v$ and $z$, and the input torque $T_{\mathrm{e}}$ are available for the state estimation.

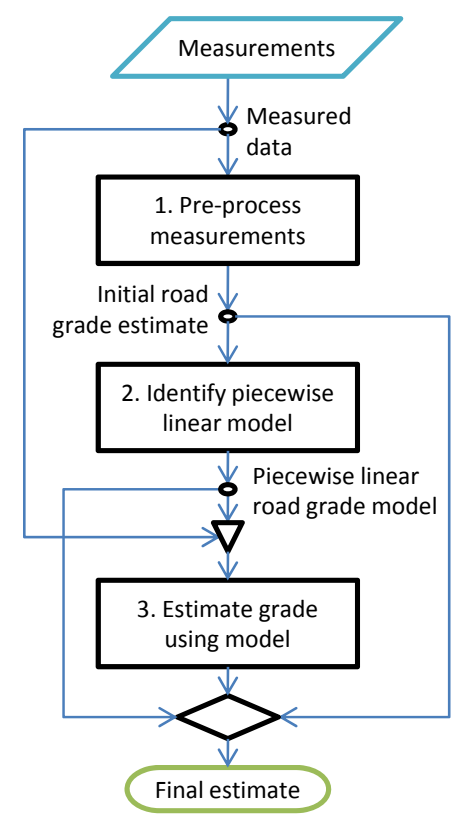

Fig. 3. In step 1 measurements from a road section are used to generate a first road grade estimate. In step 2, a piecewise linear road grade model is identified from the initial estimate. The estimation method in step 3 uses the piecewise linear model and measured data to produce a final estimate. Depending on the application requirements and chosen parameters either of the produced estimates may be chosen as the output of the process.

\section{State Estimation}

The states in the systems models with and without modeled changes in the road grade are estimated using an extended Kalman filter. The true time-varying measurement and process noise covariance matrices $R_{k}$ and $Q_{k}$ are not known, instead they are used as design parameters to minimize the estimation error, based on training data. Different values for $Q_{k}$ were used with each of the models $\dot{\alpha}_{i}(s)=0$ and $\dot{\alpha}_{i}(s)=c_{i}, i=1, \ldots, N$, respectively.

If the current road segment has been driven earlier the estimated error covariance is used together with the estimated covariance of the currently stored road grade estimate based on all previous passes over the same road section to update the stored estimate. This data fusion procedure is distributed in time in the sense that a new road grade estimate can be obtained after each new pass over a road section without access to all previous measurements. A detailed account of the estimation method can be found in [7].

\section{E. Road Grade Estimation Method}

A road grade estimation method that exploits the piecewise linear nature of the road grade signal through a three step process is proposed. The method is illustrated in Figures 3 and 4. Figure 3 describes the work flow involved in transforming measurements into a final road grade estimate, and Figure 4 illustrates each of the steps for an example road section. In the first step the measurement data is preprocessed to obtain a first road grade estimate on which to base the identification of linear segments. Second, the first estimate of the road grade is used to identify a piecewise 


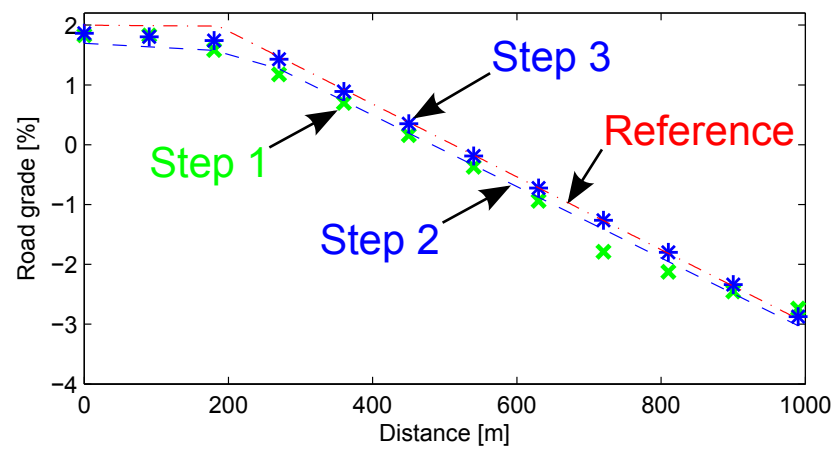

Fig. 4. The output of step 1, an initial road grade estimate is illustrated by a series of $(x)$ symbols. The linear road grade model obtained in step 2 is also shown (dashed). The final road grade estimate from step 3 is shown as (*). A piecewise linear model based on the reference road grade is included for comparison (dash-dotted).

linear model. In the third step the piecewise linear model is used to find a final road grade estimate. As illustrated by the final decision point below the third step in the process flow diagram the output of either of the steps could be used as a road grade estimate. The choice of how many processing steps to apply depends on the application.

This new process is demonstrated and evaluated in this paper with implementations for each of the steps. The performance of the proposed method is compared to a previous method, based on real world measurements. The first step in the process is completed through the use of this previous road grade estimation method, which does not require any model for the road grade signal. The second step is completed through optimization of the knot points of a least-squares optimal linear spline. In the third step a novel version of the method from step one, which uses the identified piecewise affine road grade model, is applied. This novel method uses the system model described in Section II-C.

\section{EXPERIMENTAL RESULTS}

The proposed road grade estimation method has been implemented and applied to real world measurements recorded south of Södertälje, Sweden. The results presentation consists of the experimental setup, an analysis of the modeling error caused by neglecting to model road grade changes, and estimation results obtained by using the proposed three step road grade estimation method to estimate the road grade based on collected data.

The end result of the proposed method is an estimated road grade based on all the measurements collected along a specific road section, at all occasions the vehicle has passed this specific section. As an example the final estimated road grade for a short section is shown in Figure 5. The new method with a piecewise linear road grade model is compared to a previous method described in [7]. These two methods will be referred to as the proposed and previous methods. The proposed method generally performs better than the previous method, with an average reduction in the root mean square error (rmse) in the estimation of $6-15 \%$

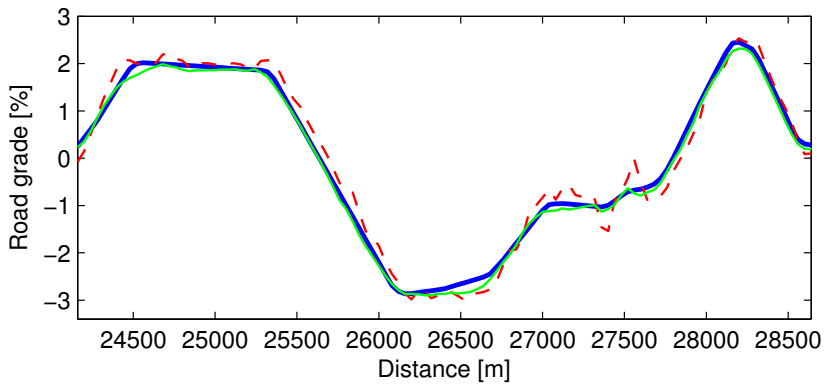

Fig. 5. A road grade estimate from the proposed method (solid) is shown together with an estimate based on a previous method (thin solid) and a reference road grade measurement (thin dashed). The performance improvement with the new proposed method is a $16 \%$ reduction in the rmse compared to the reference for the left half of the section, and a $1 \%$ reduction in the average rmse for the right half. The new method is generally better than, or at worst, equal to the previous method in estimation performance.

depending on the discretization step length.

\section{A. Experiment setup}

The proposed road grade estimation algorithm has been evaluated using data obtained from a $34 \mathrm{~km}$ section in the southbound direction, and a $43 \mathrm{~km}$ section in the northbound direction of highway E4 south of Södertälje. Data were gathered using two vehicles in a total of ten experiments, five in the southbound direction and five in the northbound direction along the test road. Most of the signals needed for the road grade estimation are available on the CAN bus of stock production trucks. These are the vehicle speed, engine torque (calculated based on fuel injection times), current gear, gearshift status, and brake utilization. The CAN bus signals were recorded using a laptop. There was no GPS data available on the vehicle bus, instead an external single frequency standard positioning service VBOX GPS receiver with a CAN interface was used.

The absolute position obtained from the GPS was used to synchronize data from the different measurements. The recorded data were divided into 15 sections of equal length, for each of the directions of travel. The results were analyzed both for each section individually, and as averages for all 30 sections.

\section{B. Prediction error}

The influence of the road model depends on the sampling step size employed, and the assumptions on the process and noise variance models. Assuming a smaller variance for the process noise increases the effect of the road model. Increasing the distance between samples also increases the effect of road grade model assumptions, as the prediction is carried out over a longer distance. The grade of a true road with the same rate of change in the road grade will thus have changed more when the next sample arrives, and the ratio of samples per unit of prediction error decreases.

Figure 6 shows the prediction error that will result from adopting a model in which the road grade doesn't change (i.e., $\dot{\alpha}=0$ ), when the real road has a vertical curve radius $R$ 

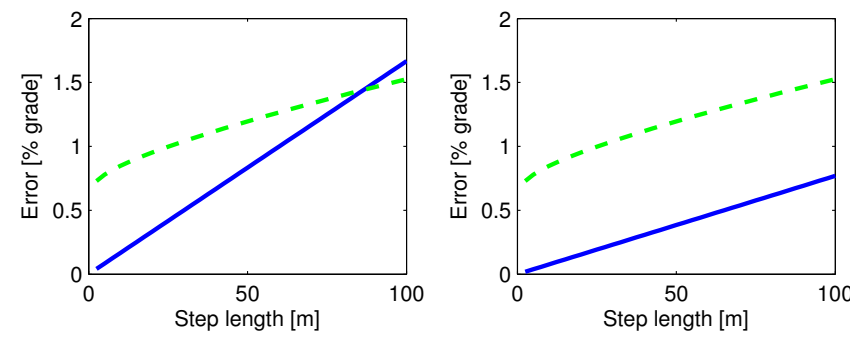

Fig. 6. The prediction error resulting from adopting the model $\dot{\alpha}(s)=0$ when the true model is $\dot{\alpha}(s)=1 / R=1 / 6000$ (left) or $\dot{\alpha}(s)=1 / R=1 / 13000$ (right) is shown as a function of the sample distance (solid). Also included is the expected standard deviation of the one step ahead prediction error for the Kalman filter used for state estimation (dashed). The importance of the model choice increases for larger step sizes.

designed according to the SRA guidelines in Table I for the reference speeds $90 \mathrm{~km} / \mathrm{h}$ (left figure) and $110 \mathrm{~km} / \mathrm{h}$ (right figure). It is compared to the expected prediction standard deviation of the proposed road grade estimation method. The computation is based on the system model given in Section II, when the vehicle operates at a constant speed of $80 \mathrm{~km} / \mathrm{h}$. For longer sampling times the modeling error becomes increasingly large compared to the expected prediction standard deviation. The real influence of the prediction error is however limited by the fact that large parts of the test road have very small changes in the road grade. When the road grade changes slowly the assumption $\dot{\alpha}=0$ is quite accurate.

\section{Experimental results}

The estimation results are evaluated based on the rmse in the estimated road grade. Since five measurements are available at each location along the road, and a key feature of the proposed method is the ability to use multiple measurements at the same location, the estimation errors based on all the available data are compared.

The distance sample step length used when estimating the grade affects many factors that influence the quality of the road grade estimate. An increased step length will for example decrease the influence of the altitude measurement noise in the GPS on the numerical derivative. Increasing the step length also makes vehicle and road grade model errors more important, as the ratio of samples per unit of prediction error decreases. Finally, the error from the zeroorder hold assumption when sampling data for the model based predictions also increases with increased step size. The experimental results have therefore been evaluated both at the full sample rate provided by the sensors, a distance step of $2.5 \mathrm{~m}$, and at increasing step sizes up to $100 \mathrm{~m}$. Input data for a step size $2.5 \cdot n \mathrm{~m}$ has been obtained by simply keeping only every $n$ :th measurement. Since a total of 30525 sample points have been used for $76 \mathrm{~km}$ of road the statistical errors introduced by this process are minimal.

It is of interest to relate the performance of the proposed method to the quality of the measurements. Since the desired quantity, the road grade, cannot be measured directly using

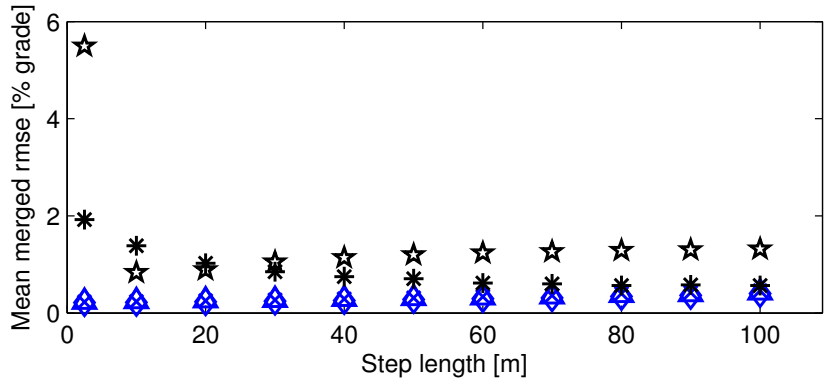

Fig. 7. The proposed road grade estimation method is compared to directly computing the road grade based on the measured data. Since five measurements are available at each location along the road, the directly computed results are averaged before the average rmse for all the studied sections along the test road is calculated as a function of the discretization step size. The resulting rmse from the GPS measurements is denoted by $(*)$ The measurement noise from the GPS causes large errors in the numerical derivative for short step lengths. For very large step lengths the estimate is

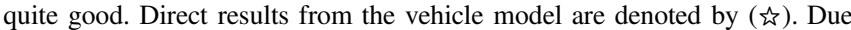
to measurement noise the estimate is bad for the smallest step size. For large step sizes the zero-order hold discretization error causes bad performance. The results after each of the steps of the proposed method are similar on the scale of this figure. The result of the previous method, which is identical to the result after step one of the proposed method, is denoted by $(x)$. The rmse of the linear model obtained in step two is denoted by $(\Delta)$. The final result of the proposed new method is denoted by $(\diamond)$.

the available sensors two direct unfiltered estimates are used instead. These are based on the vehicle model and the GPS altitude. For the vehicle model the road grade is found based on the speed change prediction (2), the measured speed change between two samples, the measured engine torque and relevant vehicle parameters. For the GPS the numerical derivative, based on a one step secant approximation, of the altitude change is used as a road grade estimate. The absolute road grade rmse's after each of the three steps of the proposed estimation method have been completed are shown in Figure 7, together with the rmse's for the two estimates directly based on the data at each consecutive pair of sample points. It is clear that a major improvement occurs in the first filtering step for all step sizes. Using the piecewise linear nature of the road grade signal by applying steps two and three further improves the estimate, particularly for the larger step sizes. This can be seen more clearly in Figure 8.sec:conclusions

The experiments show that the proposed new method generally decreases the rmse's relative the previous method. As predicted above the improvement grows with increasing step size, and for the smallest studied step sizes the improvement in this study is small, or in a few cases even negative. The performance of the estimation method with the piecewise linear road grade model compared to that of the previous method as a function of step size is shown in Figure 8.

The results also show that the performance of the piecewise linear model varies considerably between sections of the test road. Figure 9 shows the performance change with the new method for each analyzed section. This result can be explained by the deviations from the assumed model in the reference road grade profile. In some sections there 

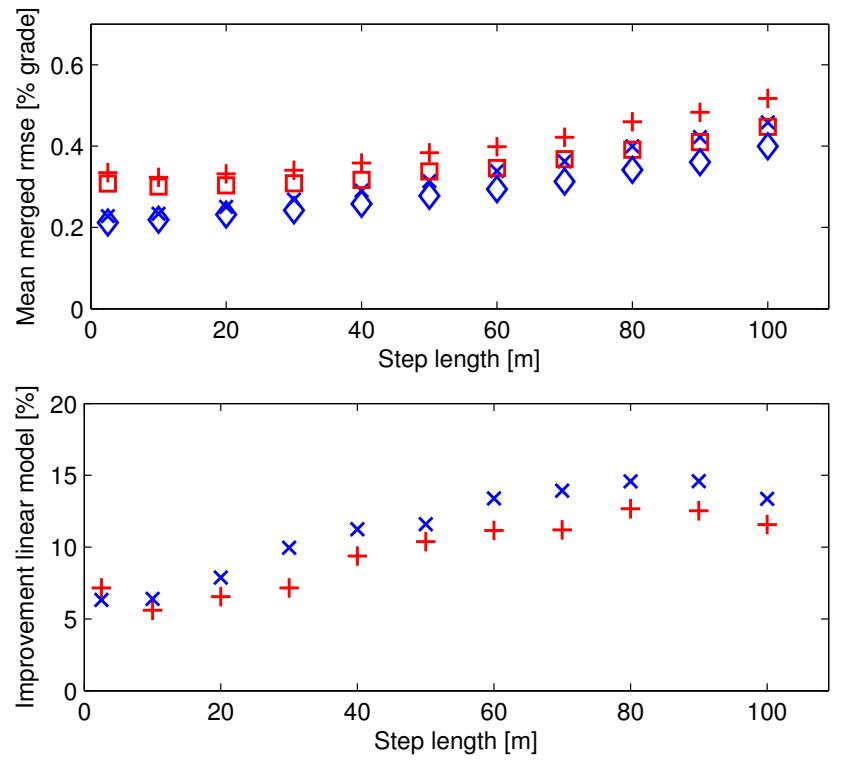

Fig. 8. A larger distance between sample points increases the model induced error when the road grade changes quickly. This can be seen as an increase in the performance gain for larger step sizes when the piecewise linear road grade model is used. The average road grade rmse's for the investigated step lengths for the previous method $(+)$ and the new method ( $\square$ ) are shown together with the road grade rmse's for the fused estimates The fused error is denoted by $(\times)$ for the previous method and $(\diamond)$ for the new method with the linear model (top figure). The relative improvement in percent, as an average for the individual experiments and for the fused estimates is also shown as a function of the step length. The average improvement for the individual experiments is denoted by $(+)$, and the improvement in the fused estimate by $(\times)$ (bottom figure).

are obvious features in the reference profile that are not captured by the linear segmentation of measurement data. Since the piecewise linear model carries a higher trust in the Kalman filter than the model predicting no change used in the previous method, larger errors may result when short term peaks that are not included in the piecewise linear model occur.

This study gives a clear indication that it may be worthwhile to investigate methods to automatically segment a road to obtain a piecewise linear model before making the final road grade estimation. Due to the performance differences between the experiments and step lengths, and the increased computational load from the segmentation, the application has to be carefully evaluated in order to make a choice between the previous and the new methods.

\section{CONCLUSIONS AND FUTURE WORKS}

Predictive HDV speed control strategies rely on high quality stored road grade information. Such information for future use can be obtained while driving, by estimation of the current road grade with standard mounted sensors. The proposed estimation method, using a piecewise linear road grade model, reduces the mean rmse of the final merged road grade estimates by $6-15 \%$ depending on the sample distance. The performance gain grows with the step size, as
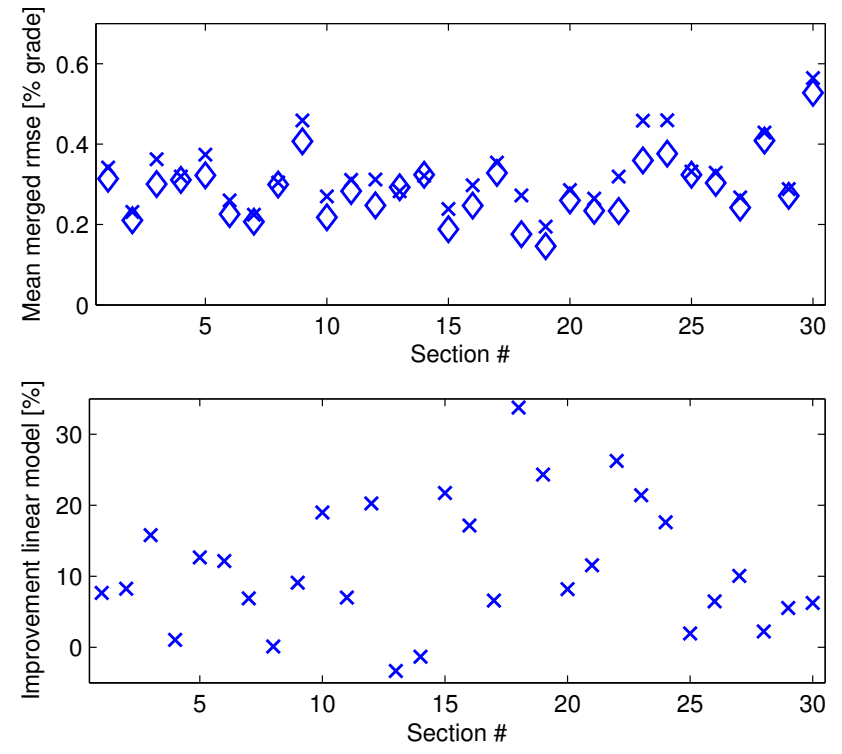

Fig. 9. The road grade rmse for the fused estimate for each road section based on all experiments is denoted by $(\times)$ for the previous method and $(\diamond)$ for the new method (top figure). The relative rmse decrease in percent from applying the new method is denoted by $(x)$ (bottom figure).

is expected due to the reduced density of data points available to correct erroneous model based predictions in the Kalman filter.

The main open question that needs to be solved to make the method useful in practice is the segmentation issue. The short test sections used herein can relatively easily be dealt with in their entirety when finding knot points and linear segments, using non-linear optimization. For longer road sections some other method will be needed.

\section{REFERENCES}

[1] E. Gaeke, "Road grade sensor," US Patent \#3,752,251, 1973, filing date: Aug 17, 1971 Issue date: Aug 1973 Inventor: Edward G. Gaeke Assignee: General Motors Corporation.

[2] H. Bae, J. Ruy, and J. Gerdes, "Road grade and vehicle parameter estimation for longitudinal control using GPS," in Proceedings of IEEE Conference on Intelligent Transportation Systems, San Francisco, CA, 2001.

[3] P. Lingman and B. Schmidtbauer, "Road slope and vehicle mass estimation using Kalman filtering," in Proceedings of the 17th IAVSD Symposium, Copenhagen, Denmark, 2001.

[4] A. Vahidi, A. Stefanopolou, and H. Peng, "Recursive least squares with forgetting for online estimation of vehicle mass and road grade: Theory and experiments," Journal of Vehicle System Dynamics, vol. 43, pp. 3157, 2005.

[5] H. Fathy, D. Kang, and J. Stein, "Online vehicle mass estimation using recursive least squares and supervisory data extraction," in Proceedings of American Control Conference, Seattle, Washington, USA, 2008.

[6] P. Sahlholm, "Iterative road grade estimation for heavy duty vehicle control," Royal Institute of Technology (KTH), Tech. Rep. TRITA-EE 2008:056, November 2008, Licentiate Thesis.

[7] P. Sahlholm and K. H. Johansson, "Road grade estimation for lookahead vehicle control using multiple measurement runs," Control Engineering Practice, vol. In Press, Corrected Proof, pp. -, 2009.

[8] U. Kiencke and L. Nielsen, Automotive Control Systems. Springer Verlag, Berlin, 2003.

[9] Vägars och gators utformning, VGU [Road and street design], 2004th ed., Vägverket, Sektion Utformning av vägar och gator, Vägverket, Butiken, SE-781 87 Borlänge, Sweden. 